to be submitted to Phys. Rev. C.

\title{
High-energy pion-nucleus elastic scattering
}

\author{
C. M. Chen \\ Los Alamos National Laboratory, Los Alamos, NM 87545 \\ and \\ Department of Physics and Center for Theoretical Physics, \\ Texas AEM University, College Station, TX 77843 \\ D. J. Ernst \\ Department of Physics and Center for Theoretical Physics, \\ Texas A $6 M$ University, College Station, TX 77843 \\ and \\ Department of Physics and Astronomy, Vanderbilt University, Nashville, TN 37235 \\ Mikkel B. Johnson \\ Los Alamos National Laboratory, Los Alamos, NM 87545
}

(Received 21 December 1992)

We investigate theoretical approaches to pion-nucleus elastic scattering at high energies $\left(300 \leq T_{\pi} \leq 1 \mathrm{GeV}\right)$. A "model-exact" calculation of the lowest-order microscopic optical model, carried out in momentum space and including the full Fermi averaging integration, a realistic off-shell pion-nucleon scattering amplitude and fully covariant kinematics, is used to calibrate a much simpler theory. The simpler theory utilizes a local optical potential with an eikonal propagator and includes the Coulomb interaction and the first Wallace correction, both of which are found to be important. Comparisons of differential cross sections out to beyond the second minimum are made for light and heavy nuclei. Particularly for nuclei as heavy as 
${ }^{40} \mathrm{Ca}$, the eikonal theory is found to be an excellent approximation to the full theory.

PACS number(s): 25.80.Ek, 24.10.Eq, 24.10.Jv

Typeset Using REVTEX 


\section{INTRODUCTION}

The pion-nucleus interaction on and below the $\Delta_{33}$ resonance has been extensively studied, and microscopic models of elastic scattering do a reasonable job [1] of describing the data. The pion-nucleus interaction at energies above the $\Delta_{33}$ resonance has received [2, 3, 4, 4, 5] much less attention. In this energy region $\left(300 \mathrm{MeV} \leq T_{\pi} \leq 1 \mathrm{GeV}\right)$, the pion has a much shorter wavelength. For example, the wavelength at resonance is about $4 \mathrm{fm}$ (about the size of the nucleus) while at $1 \mathrm{GeV}$ the wavelength is $1 \mathrm{fm}$ (about the size of a single nucleon). The shorter wavelength implies that elastic and inelastic data at the higher energies will provide sensitivity to the details of the spatial dependence of the ground-state and transition

densities. The data may also prove sensitive to modifications [6,7] of the properties of the nucleon in the nuclear medium, or reveal significant contributions from exchange currents 81.

Moreover, the pion-nucleon two-body interaction becomes much weaker as one goes to energies above the $\Delta_{33}$ resonance. The two-body total cross section becomes less than 30 mb, which is about $15 \%$ of that on the $\Delta_{33}$ resonance. The first implication of this weaker amplitude is that the pion is able to penetrate deeper into the nucleus. A simple estimate (9) from total reaction cross section studies shows that a projectile can penetrate into a target to a radius that is equal to the impact parameter at which the profile function equals one mean free path. In Fig. 11 the profile function for ${ }^{12} \mathrm{C},{ }^{40} \mathrm{Ca}$ and ${ }^{208} \mathrm{~Pb}$ are pictured, and the arrows indicate approximately how far into a nucleus a pion of the labeled energy can penetrate. The pion in the energy region from $500 \mathrm{MeV}$ to $1 \mathrm{GeV}$ is one of the most penetrating of the strongly interacting particles.

The second implication of the weaker two-body cross section is that multiple scattering theory for the optical potential becomes increasingly convergent. A simple estimate of the convergence is obtained by comparing a typical second-order term in the optical potential to the first-order term. For the case of short-range correlations, one obtains [10]

$$
R \equiv \frac{U^{(2)}}{U^{(1)}}=\sqrt{\sigma} \frac{\ell_{c}}{k} \rho
$$


where $\sigma$ is the total two-body cross section, $\ell_{c}$ is the correlation length, and $\rho$ is the nuclear density at which the pion interacts (See Fig. 1). The factor of $1 / k$, where $k$ is the incident pion momentum, reflects the suppression of the pion propagator in $U^{(2)}$ with increasing energy. On resonance, we find $R \approx 0.1$, with $R<1$ only because $\rho$ is so small there. At $500 \mathrm{MeV}$, we find $R \approx 0.04$ and at $1 \mathrm{GeV}, R \approx 0.02$. Thus differences between the data and a carefully calculated result utilizing a first-order optical potential would be a strong indication of the presence of unconventional phenomena such as mesonic current contributions, modified nucleon properties in the medium, or other yet to be thought of effects.

One option for calculating high-energy pion scattering is a momentum-space opticalpotential approach. This presents the opportunity [11] to calculate the scattering from a lowest-order optical potential including the following features 1.) exact Fermi-averaging integration, 2.) fully covariant kinematics [12], normalizations, and phase-space factors, 3.) invariant amplitudes [11,13] and 4.) finite-range, physically motivated two-body off-shell [13] amplitudes. Within the multiple scattering theory developed in Refs. [1, [14] this is an exact calculation of the lowest-order optical potential. A brief review of this approach will be given in Sec. 四.

A practical difficulty arises when one wants to use the momentum-space optical model to study high-energy pion-nucleus scattering. As the projectile energy increases, more partial waves are needed in the pion-nucleon two-body amplitudes. Below $300 \mathrm{MeV}$, only S-waves and $\mathrm{P}$-waves are needed. Above $300 \mathrm{MeV}, \mathrm{D}$-waves become important. F-waves become significant above $500 \mathrm{MeV}$ and $\mathrm{G}$-waves and $\mathrm{H}$-waves above $700 \mathrm{MeV}$. At the same time the number of pion-nucleus partial waves is increasing at a rate proportional to the pion momentum. At the high energies the momentum-space approach becomes prohibitively computer-intensive, and one would like to search for a simpler alternative.

The semi-classical theory immediately comes to mind as an alternative to the momentum-space approach. It is often used at high energies not only because it is easier to compute, but also because the simpler character of the theory facilitates obtaining 
physical insights into the reaction. However, the semi-classical theory is expected to be a good approximation only for local potentials and only when the wavelength is sufficiently short. Because the exact pion-nucleus optical potential appears to be highly nonlocal, we examine the role that nonlocalities play in high-energy pion-nucleus scattering. This is done numerically in momentum space and is presented in Sec. ПA. We undertake this study as a first step in obtaining a more quantitative measure of the validity of the semi-classical theory than currently exist in the literature.

In Sec. [IB we present our semi-classical model [15]. In order to have a quantitative as well as a simple model for confronting data, we include the Coulomb interaction and the first Wallace correction [16]. We establish the validity of the semi-classical model both by examining the size of the Wallace correction and by comparing it to the model-exact results obtained in momentum space. Comparisons of the eikonal model to model-exact calculations are made in Sec. [II].

A previous similar investigation can be found in Ref. [17]. There a factorized approximation [18] was used in the momentum-space calculations, so that the full nonlocality of the optical potential was not considered. Furthermore, Glauber multiple scattering theory [19 was proposed, whereas we will examine an even simpler model, that of a local optical potential with the scattering solved via an eikonal approximation. A more detailed discussion that compares and contrasts our results with previous work is given in Sec. IV. A summary, conclusions and future prospects are presented in Sec. \.

\section{SCATTERING THEORY}

At the higher-energies where the pion-nucleon amplitude becomes a smooth function of energy and is much weaker than on resonance, the first-order optical potential may well be adequate for describing all of the conventional nuclear physics phenomena that enter into the reaction dynamics. Whether this is so remains for further investigation. Independent of this question, there is the question of whether simpler approaches to the calculation of the lowest- 
order optical potential and the scattering from this potential are reasonably quantitative. It is this question upon which we concentrate here. Below we first review the momentumspace optical potential which serves as our 'model-exact' calculation. In momentum-space, we examine the importance of the nonlocalities caused by both the resonance propagation and the finite range of the two-body amplitude. Finding that these both can be accurately approximated, we present a simple eikonal model.

Both the optical and the eikonal models use the same target wave functions, which are obtained from Hartree-Fock calculations [20,21] with spurious center-of-mass motion removed as discussed in A. They also use the same on-shell pion-nucleon two-body amplitudes, which are constructed from Arndt's [22] and Höhler's [23] phase-shifts.

\section{A. Optical potential}

A complete description of the momentum-space optical potential that we use can be found in Ref. [11], and a detailed discussion of the covariant kinematics that we use can be found in Refs. [12]. The formal multiple-scattering theory in which this work is embedded is given in Refs. [1,14. Here we provide only a brief overview.

The first-order optical potential in the impulse approximation can be written as,

$$
\begin{aligned}
\left\langle\vec{k}_{\pi}^{\prime} \vec{k}_{A}^{\prime}\left|U_{\mathrm{mo}}(E)\right| \vec{k}_{\pi} \vec{k}_{A}\right\rangle=\sum_{\alpha} \int \frac{d^{3} k_{A-1}}{2 \bar{E}_{A-1}} \frac{d^{3} k_{N}^{\prime}}{2 \bar{E}_{N}^{\prime}} \frac{d^{3} k_{N}}{2 \bar{E}_{N}}\left\langle\Psi_{\alpha, \vec{k}_{A}^{\prime}}^{\prime} \mid \vec{k}_{N}^{\prime} \vec{k}_{A-1}\right\rangle \\
\quad \times\left\langle\vec{k}_{\pi}^{\prime} \vec{k}_{N}^{\prime}|t(E)| \vec{k}_{\pi} \vec{k}_{N}\right\rangle\left\langle\vec{k}_{N} \vec{k}_{A-1} \mid \Psi_{\alpha, \vec{k}_{A}}\right\rangle,
\end{aligned}
$$

where $\vec{k}_{\pi}, \vec{k}_{N}$ and $\vec{k}_{A-1}$ are the momenta of the pion, the struck nucleon and the $A-1$ residual nucleons in the pion-nucleus center-of-momentum frame respectively. $E$ is the incident energy of the pion and the nucleus (in the pion-nucleus center-of-momentum frame also), and $\alpha$ is a set of quantum numbers that specifically label the nuclear bound state.

The target wave function $\left\langle\vec{k}_{N} \vec{k}_{A-1} \mid \Psi_{\alpha, \vec{k}_{A}}\right\rangle$ defined covariantly [1] contains a momentumconserving delta function $\delta\left(\vec{k}_{A}-\vec{k}_{N}-\vec{k}_{A-1}\right)$ and is a function of the relative momentum between the nucleon and the $A-1$ residual nucleons. The pion-nucleon t-matrix similarly 
contains a momentum-conserving delta function and is a function of the relative momentum between the pion and the nucleon, $\vec{\kappa}$,

$$
\left\langle\vec{k}_{N}^{\prime} \vec{k}_{\pi}^{\prime}|t(E)| \vec{k}_{N} \vec{k}_{\pi}\right\rangle=\delta\left(\vec{k}_{N}^{\prime}+\vec{k}_{\pi}^{\prime}-\vec{k}_{N}-\vec{k}_{\pi}\right)\left\langle\vec{k}^{\prime}\left|t\left[\omega_{\alpha}\left(E, \vec{k}_{\pi}, \vec{k}_{N}\right)\right]\right| \vec{\kappa}\right\rangle
$$

One of the these three momentum-conserving delta functions leads to overall momentum conservation; the others allow one to perform two of the three integrals in Eq. 2. The remaining integral is the Fermi averaging integration which must be performed numerically. The details of how we perform the integration can be found in [11].

The energy at which one evaluates the two-body t-matrix, $\omega_{\alpha}\left(E, \vec{k}_{\pi}, \vec{k}_{N}\right)$, must be carefully chosen [24] if a convergent perturbation theory is to result for calculations at and below the $\Delta 33$ resonance region. The energy $\omega_{\alpha}$ is defined covariantly by first defining the energy available to the pion-nucleon two-body subsystem

$$
E_{\pi N}=E-\sqrt{\left(\vec{k}_{\pi}+\vec{k}_{N}\right)^{2}+m_{A-1}^{2}}
$$

and then defining the invariant center-of-momentum energy for this system

$$
\omega_{\alpha}^{2}=E_{\pi N}^{2}-\left(\vec{k}_{\pi}+\vec{k}_{N}\right)^{2}
$$

The mass of the $A-1$ system, $m_{A-1}$, differs from the mass of the $A$-body target, $m_{A}$, by a nucleon mass and a binding energy, $m_{A}=m_{A-1}+m_{N}+E_{b}$. This energy must then be shifted by a 'mean-spectral energy', $E_{m s}$, which is a calculated number [24] that approximately accounts for the interaction of the intermediate $\Delta_{33}$ (or the intermediate nucleon or other hadronic resonance) with the residual nucleus. This first-order potential produces results for energies near the $\Delta_{33}$ resonance that are in remarkable agreement with the data. This is because [1] the sum of the second-order effects (Pauli exclusion, true absorption and correlation corrections) cancel amongst themselves. The use of invariant normalizations, invariant phase space and invariant amplitudes produces the phase-space factors that are present in Eq. 2.

The optical potential so defined is then inserted into the Klein-Gordon equation which is solved numerically to produce our model-exact calculation. 


\section{B. Eikonal model}

The eikonal model we propose to examine results from first replacing the optical potential of Eq. 2 by a local potential and then solving for the scattering amplitude arising from the use of the local potential and the eikonal propagator. In order to include the Coulomb interaction, we divide the scattering amplitude as

$$
F(q)=F_{p t}(q)+F_{C N}(q)
$$

where $q$ is the momentum transferred to the pion, $\vec{q}=\vec{k}_{\pi}^{\prime}-\vec{k}_{\pi}, F_{p t}$ is the point Coulomb scattering amplitude, and $F_{C N}$ is the scattering amplitude calculated from the sum of the strong nuclear potential plus a Coulomb correction, which is the difference between the point Coulomb interaction and the Coulomb interaction of a finite charge distribution. The eikonal approximation gives for $F_{C N}(q)$

$$
F_{C N}(q)=i k_{\pi} \int_{0}^{\infty} b d b J_{0}(q b) e^{i \chi_{p t}(b)} \Gamma_{C N}(E, b)
$$

where $k_{\pi}$ is the momentum of the incident pion in the pion-nucleus center-of-momentum frame, $b$ is the impact parameter, $\chi_{p t}$ is the point Coulomb phase (in the eikonal approximation), and $\Gamma_{C N}$ is the profile function defined below.

In order to incorporate the distortion and energy shift caused by the Coulomb interaction, we follow the prescription given in Ref. [25] and write the profile function as

$$
\begin{aligned}
\Gamma_{C N}(E, b) & =1-\exp \left\{i \chi_{C N}(E, b)\right\} \\
& =1-\exp \left\{i \chi_{N}\left[E, b\left(1+E V_{c}(b) / k_{\pi}^{2}\right)\right]+i \chi_{c}(b)-i \chi_{p t}(b)\right\},
\end{aligned}
$$

where $V_{c}$ is the Coulomb potential of a uniformly charged sphere, $\chi_{c}$ is the phase shift caused by that potential, and the nuclear phase shift $\chi_{N}$ is written as

$$
\chi_{N}(E, b)=-\frac{1}{2 k_{\pi}} \int_{-\infty}^{\infty} d z U_{\mathrm{eik}}\left[E-V_{c}(r), r\right],
$$

where 


$$
r=\left(b^{2}+z^{2}\right)^{1 / 2}
$$

and $U_{\text {eik }}$ is the strong potential obtained as follows. First we construct a local potential $U_{o}$ from the pion-nucleon two-body scattering amplitude and the target density by

$$
U_{o}(E, r)=-4 \pi Z\left[f_{p}(0) \rho_{p}+\frac{f_{p}^{\prime}(0)}{2 k_{\pi}^{2}} \nabla^{2} \rho_{p}\right]-4 \pi N\left[f_{n}(0) \rho_{n}+\frac{f_{n}^{\prime}(0)}{2 k_{\pi}^{2}} \nabla^{2} \rho_{n}\right],
$$

where $Z$ and $N$ are the proton number and the neutron number respectively. The scattering amplitude $f_{p}(0)\left[f_{n}(0)\right]$ is the pion-proton [pion-neutron] scattering amplitude in

the forward direction in the pion-nucleus center-of-momentum frame. The finite range of the pion-nucleon interaction is included through lowest nonvanishing order and produces the additional terms in Eq. 11 which are proportional to the derivatives of the scattering amplitude as shown in Appendix B.

In order to investigate the importance of the lowest-order corrections to the eikonal propagator as derived by Wallace [16], next we replace the potential $U_{o}(E, r)$ by the potential

$$
U_{\text {eik }}(E, r)=U_{o}+\frac{U_{o}^{2}}{4 k_{\pi}^{2}}\left(1+\frac{2 b^{2}}{r} \frac{d}{d r} \ln U_{o}\right) .
$$

The details of the derivation of this correction and higher order corrections can be found in Ref. [16].

\section{RESULTS}

In this section we investigate the importance of some of the ingredients of our modelexact calculation of high-energy pion-nucleus scattering. We will then examine how well our simple eikonal theory reproduces the results of the model-exact theory.

\section{A. Contributions from nonlocalities in the optical potential}

The most difficult part of evaluating the optical potential in the model-exact theory is performing the Fermi-averaging integration. This is known to be very important in the 
region of the $\Delta_{33}$ resonance, where proper accounting for the recoil and propagation of the pion-nucleus resonance requires a careful treatment of the dependence of the energy $\omega_{\alpha}$ in Eq. 5 on the momentum of the nucleon. Resonance propagation manifests itself as a nonlocality in the optical potential, and this represents the first source of nonlocality whose importance we want to assess for high-energy pions.

To investigate this we calculate elastic scattering of $\pi^{-}$from ${ }^{40} \mathrm{Ca}$ first with full Fermi averaging and then utilizing a closure approximation. The closure approximation that we use results from taking

$$
\omega_{\alpha}^{2}=W_{\pi N}^{2}-k_{\pi}^{2} \text { and } W_{\pi N}=E-\sqrt{k_{\pi}^{2}+m_{A-1}^{2}}
$$

This is equivalent to setting the momentum of the struck nucleon to zero in the pion-nucleus c.m. frame.

On the delta resonance, it has been found [26,27,28] that the closure approximation is totally inadequate and even the 'optimally-factorized' approximation [28] is not quantitative. At these higher energies the conclusion is not á priori clear. The pion-nucleon amplitude is, partial wave by partial wave, rather energy dependent as the individual partial waves are resonant. The total amplitude, however, exhibits only two very broad smooth peaks on a large background.

The resulting differential cross sections for pions at $500 \mathrm{MeV}$ scattering from ${ }^{40} \mathrm{Ca}$ are shown in Fig. 2. We see that the correct treatment of the recoil of the two-body pion-nucleon system only affects the depth of the minimum but not to a very significant degree.

The behavior of the pion-nucleon scattering amplitude off-shell is taken from the doorway-resonance model of Ref. [13]. Amplitudes which are resonant are separable in their dependence on the relative momenta, hence maximally nonlocal. This is the second source of nonlocality whose importance we want to investigate. In the doorway-resonance model, in each angular momentum, spin and isospin channel,

$$
\left\langle\kappa^{\prime}\left|t_{J L I}\left(\omega_{\alpha}\right)\right| \kappa\right\rangle=\frac{v\left(\kappa^{\prime}\right)}{v\left(\kappa_{o}\right)}\left\langle\kappa_{o}\left|t_{J L I}\left(\omega_{\alpha}\right)\right| \kappa_{o}\right\rangle \frac{v(\kappa)}{v\left(\kappa_{o}\right)},
$$


where $\kappa_{o}$ is the on-shell momentum, i.e. the momentum corresponding to the center-ofmomentum energy $\omega_{\alpha}$. This nonlocality, which is also factored in the delta-hole [26] model, was found quite important [28] for energies on and below the $\Delta_{33}$ resonance. For numerical convenience, we take the form factor to be a Gaussian, $v(\kappa)=\exp \left(-\kappa^{2} / \beta^{2}\right)$, and we vary $\beta$ from $500 \mathrm{MeV}$ to $4 \mathrm{GeV}$ to investigate the importance of this nonlocality. The differential cross sections for $\pi^{-}-{ }^{40} \mathrm{Ca}$ scattering calculated with this variation in the form-factor range are shown in Fig. 3. We see that the dependence on the off-shell range is weak at this energy.

There exists some confusion in the literature concerning the possible values for $\beta$. First, if one does not include invariant phase space factors explicitly, they will appear effectively in what one might wish to call the form factor. This difference is then one of semantics. However, in deciding what might be a reasonable range over which to vary the form factor, one should treat the kinematic factors explicitly. In the $P$-wave channels, an artificial and incorrect increase (in coordinate space) in the range of the form factor will also result if the pion-nucleon pole term [29] is not included in the model of the pion-nucleon amplitude. For our purposes here, the difference of interest is in examining how the cross section changes in going from the physically motivated doorway-model form factors to the higher momentum cutoff. The $4 \mathrm{GeV}$ cutoff produces an approximately zero-range interaction in coordinate space. Figure 3 demonstrates that the nonlocalities contained in a finite range interaction do not alter significantly the predicted elastic cross sections for these high energy pions.

We have found that the nonlocalities in the optical potential at the high energies do not have an important effect. This suggests that the simple eikonal approach outlined above could prove to be an adequate model for quantitative work at these energies. To investigate this, we picture the differential cross sections predicted by each model for $\pi^{ \pm \_}{ }^{12} \mathrm{C}$ and $\pi^{ \pm}-$ ${ }^{40} \mathrm{Ca}$ at $800 \mathrm{MeV} / \mathrm{c}$ in Fig. 4 and Fig. 5. The data are from Ref. [4]. As stated earlier, we construct the target density from the same wave functions as were used in the momentumspace calculation and use the same on-shell pion-nucleon scattering amplitude. For ${ }^{40} \mathrm{Ca}$, both the location of the minima and the magnitude of the cross section at the forward angles 
are in good agreement. The agreement is, however, not as quantitative for ${ }^{12} \mathrm{C}$ where the minima are slightly shifted. This difference is presumably caused in part by the fact that the kinematics of target recoil enter explicitly in the momentum-space formulation but not in the eikonal approach. The eikonal model is thus more quantitative for the heavier nuclei. We are examining the eikonal model to see if it can be further improved for the light nuclei.

\section{B. Important features in the eikonal approximation}

In this section we investigate the role of the Coulomb interaction and the first Wallace correction, which are both necessary if one wants to use the eikonal model to quantitatively approximate the results of the solution of the model-exact theory.

First, we find that the Coulomb interaction plays an important role in determining the depth of the minima in the differential cross section. To see exactly how important Coulombnuclear interference is, we compare the calculation of the $\pi^{-}-{ }^{40} \mathrm{Ca}$ differential cross section at a series of energies as shown in Fig. 6 with and without the Coulomb interaction. The Wallace correction is not included in either the solid or the dashed cureves. We find that the first minimum in the differential cross sections becomes very deep when the Coulomb interaction is included, falling well below $10^{-2} \mathrm{mb} / \mathrm{sr}$ in the region of 500 to $600 \mathrm{MeV}$. Without the Coulomb interaction, the deepest minimum is much shallower and occurs at $780 \mathrm{MeV}$. The inclusion of the Coulomb interaction in the theory is necessary if a quantitative comparison with the data for $\pi^{-}-{ }^{40} \mathrm{Ca}$ is to be made. Coulomb-nuclear interference is destructive for $\pi^{-}$and constructive for $\pi^{+}$, which produces a less dramatic effect for $\pi^{+} \_{ }^{40} \mathrm{Ca}$ scattering in this energy region.

Wallace has shown in Ref. [16] that the semi-classical approximation can be improved if higher order corrections (known as "Wallace" corrections) are included. We examine in Fig. 7 the importance of the first Wallace correction, Eq. 12, for $\pi^{-}-{ }^{40} \mathrm{Ca}$ scattering. We see in Fig. 17, in the absence of the Coulomb interaction, that the Wallace correction is dramatic on resonance, $T_{\pi}=180 \mathrm{MeV}$, where it fills in the deep minimum. At the higher energies, 
the correction interferes constructively until about $700 \mathrm{MeV}$ filling in the minima somewhat. At $780 \mathrm{MeV}$, however, the Wallace correction interferes destructively with $U_{o}$ and produce a much deeper minimum.

In Fig. 8 we turn on both the Coulomb interaction and the Wallace correction, we find a fascinating interplay between the two. Notice that at $400 \mathrm{MeV}$, for example, there two corrections are out of phase and tend to cancel. At $680 \mathrm{MeV}$, however, they are in phase and interfere destructively with $U_{o}$ producing the very deep minimum. By $780 \mathrm{MeV}$ they are both out of phase with each other but in phase with the lowest order term and thus fill in the minima. Both the Coulomb and the Wallace correction are necessary if there is to be a deep minima at $680 \mathrm{MeV}$, and not at the neighboring energies. We note that the data of Ref. [1] was taken at a pion lab momentum of $800 \mathrm{MeV} / \mathrm{c}$ which is very near $T_{\pi}=680$ $\mathrm{MeV}$. The depth of the first minimum of the differential cross section for $\pi^{-}$scattering over the energy range 400 to $800 \mathrm{MeV}$ should prove a very sensitive test of the existence, or lack thereof, of higher order corrections in the reaction dynamics.

\section{COMPARISON TO OTHER WORK}

Other work on high-energy pion-nucleus elastic scattering, which utilizes the full Glauber theory, can be found in Refs. [17,30]. In particular, a comparison between the Glauber theory and the optical model on $\pi^{ \pm}{ }^{12} \mathrm{C}$ has been made in Ref. [17] and the two approaches were found to be in good agreement at the higher energies. We earlier noted that the optical potential model there did not include the full Fermi averaging, and it was not clear to what extent the agreement found was caused by the approximate treatment of the non-localities in the optical potential. Our work clarifies that. We also note that the Glauber theory

they used is considerably more complicated than our eikonal model. To our knowledge, the Wallace corrections have not been included in any of the numerical tests of the eikonal model in this energy region. Hence, the important interplay between the Wallace and Coulomb corrections that we find has not been previously noted. We also found that the center- 
of-mass corrections in the target wave functions are important for ${ }^{12} \mathrm{C}$ but not for ${ }^{40} \mathrm{Ca}$, which is in agreement with Ref. [17]. Our work strongly supports their conclusion that pion scattering at the higher energies becomes theoretically and calculationally simpler to treat at a quantitative level.

\section{SUMMARY, CONCLUSIONS, AND FUTURE PROSPECTS}

We have compared the scattering from the fully microscopic and nonlocal lowest-order optical potential to the scattering from a simple local potential in the eikonal approximation. We have investigated the importance of several features of the full model. Within the framework of our "model-exact" optical model, these include Fermi averaging and the offshell behavior of the pion-nucleon scattering amplitude. For the eikonal theory, we examine the importance of the first Wallace correction and find that this correction is responsible for a noticeable improvement of the eikonal theory in comparison to the "model-exact" theory. In all cases, the Coulomb-nuclear interference is important and must be included if one is to compare with data.

This semi-classical eikonal theory put forth here is very simple, even simpler than the Glauber theory utilized by others. It appears to be quantitatively valid at high energy, at least for the first several minima in the differential elastic cross section. This makes possible a much simpler reaction theory than has been needed at energies at and below the $\Delta_{33}$ resonance.

At high energies and/or for heavy nuclei, use of the full momentum-space optical model becomes very time-consuming and sometimes computationally impossible. In contrast, the eikonal theory is relatively simple and fast on the computer. It therefore becomes a matter of considerable practical importance to realize that the physics is faithfully reproduced by the simple version of the theory out to the position of the second minimum in the differential cross section.

An examination of the pion-nucleon cross section shows the complex interplay of reso- 
nances. Ultimately, it is interesting to explore how resonances behave in the nucleus and how the specific features of the optical potential are suited for nuclear structure studies. In this pursuit, it will be very helpful to have a simple version of the theory as developed here. We will pursue these questions in subsequent work.

The momentum space optical model has been extended to treat kaon-nucleus scattering [32]. Results show qualitative agreement with previous work [6]. Modifications to the kaonnucleon amplitudes in the nuclear medium are needed to eliminate discrepancies between the theory and the data. On the quantitative level, results from the momentum space approach give larger discrepancies between theory and data than do those from the coordinate space calculations used in Ref. [6]. Our eikonal model, which is also a coordinate space approach and been shown to be a good approximation to the "model-exact" momentum space theory for high-energy pion scattering, can also be modified to treat the kaon scattering. Results from the eikonal model may reveal the sources of the discrepancies between the momentum space and the previous coordinate space calculations. Work on the kaon-nucleus scattering utilizing the eikonal model is in progress. 


\section{APPENDIX A: CENTER-OF-MASS CORRECTIONS}

The scattering theory developed in Ref. [11] is covariant. However, to implement it covariantly would require a covariant model of the nucleus. This would clearly be overkill as the nucleus for the cases of interest is not moving relativistically. A Galilean invariant model would thus suffice. Contemporary models of the nucleus, however, are not Galilean invariant. We use Hartree-Fock models of the nucleus which contain spurious center-of-mass motion. The approach we adopt is discussed in detail in Ref. [12]. The Hartree-Fock results are fitted to elastic electron scattering. The spurious center-of-mass motion is there treated by requiring that momentum conservation holds for average values. We make the same approximation for pion-nucleus scattering.

Specifically we calculate the target wave functions from the Hartree-Fock model and then calculate the Fourier transform of the density. We require the intrinsic density, i.e. the density without the center-of-mass motion. If the wave function were a pure harmonic oscillator, then the correction could be made exactly. Here, we make the correction as if the Hartree-Fock result were exactly a harmonic oscillator. The result [31] for obtaining the intrinsic proton density $\rho_{c m}^{p}$ from the Hartree-Fock density $\rho_{H F}^{p}$ is

$$
\rho_{c m}^{p}(r)=\int d^{3} r_{1}(B \sqrt{\pi})^{-3} e^{\left(r_{1}^{2} / B^{2}\right)} \rho_{H F}^{p}\left(\left|\vec{r}-\vec{r}_{1}\right|\right)
$$

where $B=b / \sqrt{A}$ and $b$ is the usual harmonic oscillator parameter. The Hartree-Fock calculation uses a slightly different $b$ for each orbital so we use the average value in making the center-of-mass correction.

We use $\rho_{c m}^{p}$ to calculate the r.m.s. radius and compare this to the r.m.s. radius measured in electron scattering (with the finite size of the proton removed). For ${ }^{12} \mathrm{C}$ and the HartreeFock result from Ref. [21] we find that the r.m.s. radius is 2.365 fm from the Hartree-Fock calculation while it is measured to be $2.297 \mathrm{fm}$ in electron scattering. We therefore scale the b's in the Hartree-Fock calculation by .9715 (a 3\% correction) so that the correct measured r.m.s. radius is reproduced. After the scaling, we calculate the predicted electron scattering 
and find that these wave functions reproduce electron scattering data out to a momentum transfer which is larger than is encountered in pion scattering. For ${ }^{40} \mathrm{Ca}$, these corrections were already made by Negele [20] and his wave functions are consistent with the electron scattering data.

\section{APPENDIX B: FORWARD SCATTERING AMPLITUDE}

Using a Taylor expansion, we can write the forward scattering amplitude for small angles as

$$
\begin{aligned}
f(\theta) & \approx f(0)+\left.\frac{d f(\theta)}{d \cos \theta}\right|_{\cos \theta=1}(\cos \theta-1) \\
& \approx f(0)-f^{\prime}(0) \frac{\theta^{2}}{2},
\end{aligned}
$$

where

$$
\begin{aligned}
f^{\prime}(0) & =\left.\frac{d f(\theta)}{d \cos \theta}\right|_{\cos \theta=1} \\
& =\left.\sum_{l} f_{l}(0) \frac{d P_{l}(\cos \theta)}{d \cos \theta}\right|_{\cos \theta=1} \\
& =\sum_{l} f_{l}(0) \frac{l(l+1)}{2} .
\end{aligned}
$$

We further use the approximation

$$
\theta \approx \frac{q}{k_{\pi}}
$$

and the fact that the momentum transfer $q$ in momentum space corresponds to $i \nabla$ in coordinate space, to rewrite Eq. B1] as

$$
f(\theta) \approx f(0)+\frac{f^{\prime}(0)}{2 k_{\pi}^{2}} \nabla^{2}
$$

To construct a local optical potential, we multiply $f(\theta)$ by the target density $\rho$ and obtain Eq. 11.

The forward scattering amplitude $f(0)$ and its derivative $f^{\prime}(0)$ are converted from phase shifts in the pion-nucleon c.m. frame, then transferred to the pion-nucleus c.m. frame through the relation: 


$$
f(0)=\frac{k_{\pi}}{\kappa} f_{\pi N}(0)
$$

where $\kappa$ and $f_{\pi N}(0)$ denote the pion momentum and forward scattering amplitude in the pion-nucleon c.m. frame. 


\section{REFERENCES}

[1] M. B. Johnson and D. J. Ernst, Ann. Phys. (N.Y.) 219, 266 (1992); C. M. Chen, D. J. Ernst and M. B. Johnson, to appear in Phys. Rev. C.

[2] S. H. Rokni et al., Phys. Lett. B202, 35 (1988); G. E. Parnell, D. J. Ernst and D. R. Giebink, Phys. Lett. B205, 135 (1988).

[3] A. L. Williams et al., Phys. Lett. B216, 11 (1989).

[4] D. Marlow, P. D. Barnes, N. J. Colella, S. A. Dytman, R .A. Eisenstein. R. Grace, F. Takeutchi, and W. R. Wharton; S. Bart, D. Hancock, R. Hackenberg, E. Hungerford, W. Mayes, L. Pinsky, and T. Williams; R. Chrien, H. Palevsky, and R. Sutter, Phys. Rev. C 25, 2619 (1982).

[5] The User's View for the Future of LAMPF, 1989; Reports from the Pion Physics Working Group, ed. G. R. Burleson and D. J. Ernst (Los Alamos Report LA-11673-MS); D. Dehnhard and D. J. Ernst, in PILAC Users Group Report on Physics with PILAC, (Los Alamos Report LA-UR-92-150).

[6] P. B. Siegel, W. B. Kaufmann, and W. R. Gibbs, Phys. Rev. C 30, 1256 (1984); P. B. Siegel, W. B. Kaufmann, and W. R. Gibbs, Phys. Rev. C 31, 2184 (1985).

[7] G. E. Brown, C. B. Dover, P. B. Siegel and W. Weise, Phys. Rev. Lett. 26, 2723 (1988);

[8] M. F. Jiang and D. S. Koltun, to appear in Phys. Rev. C.

[9] D. J. Ernst, Phys. Rev. C19, 896 (1979).

[10] D. J. Ernst, J. T. Londergan, G. A. Miller and R. M. Thaler, Phys. Rev. C16, 537 (1977).

[11] D. R. Giebink and D. J. Ernst, Comp. Phys. Comm. 48, 407 (1988).

[12] D. J. Ernst and G. A. Miller, Phys. Rev. C21, 1472 (1980); D. L. Weiss and D. J. Ernst, Phys. Rev. C26, 605 (1982); D. R. Giebink, Phys. Rev. C25, 2133 (1982). 
[13] D. J. Ernst, G. E. Parnell and C. Assad, Nucl. Phys. A518, 658 (1990).

[14] M. B. Johnson and D. J. Ernst, Phys. Rev. C27, 709 (1983).

[15] J. Germond and M. B. Johnson, Phys. Rev. C22, 1622 (1980); J. Germond, M. B. Johnson and J. A. Johnstone, Phys. Rev. C32, 983 (1985).

[16] S. J. Wallace, Ann. Phys. (N.Y.) 78, 190 (1973); S. J. Wallace, Phys. Rev. D8, 1846 (1973); S. J. Wallace, Phys. Rev. C8, 2043 (1973).

[17] M. Arima, K. Masutani, and R. Seki, Phys. Rev. C44, 415 (1991).

[18] K. A. Eisenstein and F. Tabakin, Comp. Phys. Comm. 12, 237 (1976).

[19] T. Glauber, Lectures in Theoretical Physics (Interscience, New York, 1959), Vol. I, p. 315.

[20] J. W. Negele, Phys. Rev. C1, 1260 (1969).

[21] M. Beiner, H. Flocard, N. Van Gai and P. Quentin, Nucl. Phys. A238, 29 (1975).

[22] R. Arndt, computer code SAID, Phys. Rev. D28, 97 (1983).

[23] G. Höhler in $\Pi N$ Newsletter No. 1,, ed. G. Höhler and B. M. K. Nefkins (Karlsruhe, 1984); R. Koch, Z. Phys. C29, 597 (1985).

[24] D. J. Ernst and M. B. Johnson, Phys. Rev. C32, 940 (1985); D. J. Ernst, D. R. Giebink and M. B. Johnson, Phys. Lett. B182, 242 (1986).

[25] J. Germond and C. Wilkin, Ann. Phys. 121,285 (1979).

[26] M. Hirata, J.-H. Koch, F. Lenz, and E. J. Moniz, Ann. Phys. (N.Y.) 120, 205 (1979).

[27] L. C. Liu and C. M. Shakin, Prog. Part. Nucl. Phys. 5, 207 (1982).

[28] D. J. Ernst, G. A. Miller and D. L. Weiss, Phys. Rev. C 27, 2733 (1983).

[29] D. J. Ernst and M. B. Johnson, Phys. Rev. C17, 247 (1978).

[30] E. Oset and D. Strottman, Phys. Rev. C44, 468 (1991). 
[31] J. P. Elliot and T. H. R. Skyrme, Proc. Roy. Soc. (London) A232, 561 (1955).

[32] C. M. Chen and D. J. Ernst, Phys. Rev. C45, 2011 (1992). 


\section{FIGURES}

FIG. 1. Profile function $S(b)$ for ${ }^{12} \mathrm{C},{ }^{40} \mathrm{Ca}$ and ${ }^{208} \mathrm{~Pb}$. The arrows indicate the depth to which the pion (labeled by its energy) can penetrate.

FIG. 2. The differential cross section for elastic scattering of $\pi^{-}$from ${ }^{40} \mathrm{Ca}$ at $500 \mathrm{MeV}$ verses center-of-mass angle. The solid curve is the result of a calculation in which the full Fermi averaging is performed exactly, while the dashed curve uses the closure approximation defined in Eq. 13.

FIG. 3. The differential cross section calculated from different form factor ranges $\beta$ defined in Sec.III for $\pi^{-}-{ }^{40} \mathrm{Ca}$ elastic scattering at $500 \mathrm{MeV}$. The solid curve is from the "model-exact" theory. The doted curve corresponds to $\beta=4 \mathrm{GeV}$. The dashed curve is the result from setting $\beta=500 \mathrm{MeV}$.

FIG. 4. The elastic differential cross sections for $\pi^{ \pm \_}{ }^{12} \mathrm{C}$ scattering at a pion lab momentum $800 \mathrm{MeV} / \mathrm{c}$. The solid curves are from the momentum space calculations, while the dashed curves are from the eikonal model. Data are obtained from Ref. 朋.

FIG. 5. The same as Fig. 4 except the target is ${ }^{40} \mathrm{Ca}$.

FIG. 6. $\pi^{-}{ }^{40} \mathrm{Ca}$ elastic differential cross section calculated from the eikonal model at labeled pion lab energies. The Coulomb interaction is included in all the solid curves, but not the dashed curves. The Wallace correction is not included here.

FIG. 7. $\pi^{-}{ }^{40} \mathrm{Ca}$ elastic differential cross sections calculated from the eikonal model at labeled pion lab energies. The dashed curves are the same as in Fig. 6. The solid curves include the Wallace correction but not the Coulomb interaction.

FIG. 8. The dashes curves are the same as in Figs. 6 and 7. The solid curves now contain both the Wallace correction and the Coulomb interaction. 